\title{
Monitoring the Heat Accumulation during Fabrication of Surface Micro- patterns on Metallic Surfaces Using Direct Laser Interference Patterning
}

\author{
Nikolai Schröder ${ }^{1}$, Germán Vergara ${ }^{2}$, Bogdan Voisat $^{1}$ and Andrés Fabián Lasagni ${ }^{1,3}$ \\ ${ }^{1}$ Technische Universität Dresden, Institut für Fertigungstechnik, George-Bähr-Str. 3c, \\ 01069 Dresden, Germany \\ Corresponding author's e-mail: nikolai.schroeder@tu-dresden.de \\ ${ }^{2}$ New Infrared Technologies, Calle Vidrieros, 30 - Nave 2, Boadilla del Monte, \\ 28660 Madrid, Spain \\ ${ }^{3}$ Fraunhofer-Institut für Werkstoff- und Strahltechnik IWS, Winterbergstr. 28, \\ 01277 Dresden, Germany
}

\begin{abstract}
Metallic samples with unique micro- and nano-scale surface structures can easily be fabricated with Direct Laser Interference Patterning. Like in all laser processes, the material interacts with the laser radiation and as a result, thermal effects occur. These effects have a significant influence on the resulting quality of the surface patterns. In this study, the thermal effects occurring during Direct Laser Interference Patterning of stainless steel and aluminum sheets are investigated. The used experimental setup consisted of a picosecond pulsed laser source operating at $532 \mathrm{~nm}$ wavelength, combined with a two-beam interference optical head. An infrared camera in an off-axis position is used to detect the resulting thermal radiation of the laser process varying different process parameters such as laser power and repetition rate. The obtained results reveal a correlation between the recorded signal by the infrared camera and the reached surface quality. They show an impact of the thermal effects on the quality of the surfaces and the amount of solidified material on the resulting line-like pattern. Threshold values of the detected infrared signal detected are determined to classify the obtained surface conditions.
\end{abstract}

DOI: $10.2961 /$ jlmn.2020.02.2013

Keywords: direct laser interference patterning, DLIP, micro structuring, stainless steel, aluminum, heat accumulation, high-speed, pulsed laser, monitoring

\section{Introduction}

Smart surfaces with tailored properties have gained significant attention in the scientific community [1,2]. Nature provides a plurality of different applications based on the unrivaled periodic surface structure to increase efficiency and performance to a maximum level. Plants, animals and insects are taking advantage of these unique skills [3-5]. One of the most prominent examples from nature is the water repellent and self-cleaning puppetries of the lotus leaf. The surface of the leaf exhibits a periodic structure in the nano and micrometer range, which is responsible, together with the surface chemistry of the above above-mentioned functionalities [6].

It is known that laser technologies are outstanding in creating functional surface structures by reproducing the geometry of several natural examples [7-12]. An efficient laserbased method capable of functionalizing surfaces is Direct Laser Interference Patterning (DLIP) [13]. This technology provides a one-step solution to produce periodic micropatterns on different materials with high throughputs using either femtosecond, picosecond or nanosecond laser pulses [14]. Compared to alternative laser-based methods, DLIP offers a plurality of surface patterns with a feature sizes down to $170 \mathrm{~nm}[15,16]$. Other competing laser technologies, that have been already successfully used to produce repetitive periodic structures, are exemplarily Microsphere Laser Nano-Processing, Laser Interference Lithography, and Far-
Field Multifocal Array Nanopatterning, which permit to reach resolutions of sub-100 nm, $80 \mathrm{~nm}$ and $95 \mathrm{~nm}$, respectively [17-19]. Like in other techniques utilizing short and ultra-short laser pulses, the physical mechanisms involved in the structure formation is due to the interaction between ultrashort laser pulses and solid matter [20-22]. Thus, depending on material properties as well as the laser processing parameters, different effects can be obtained. Among the most interesting and relevant physical effects, which can be observed during the laser treatment, the thermal effects at the material surface are of high importance, since they have a significant influence on the surface quality of the processed samples [23]. In consequence, in-line monitoring strategies are necessary to ensure a certain quality of the processed parts.

Monitoring the thermal effects has been intensively studied during continuous-wave laser-based processes, like laser welding [24-29] or additive manufacturing [30-35]. In order to limit heat accumulation and avoid detrimental influences, pulsed laser systems have been proven to be highly effective. However, with increasing the power and repetition rate of a laser, the produced heat at the material by the laser process cannot be dissipated and starts to accumulate. Consequently, the process quality is afflicted under this thermal phenomenon [36]. Infrared cameras provide the opportunity to monitor the thermal effects that occur during short and 
ultra-short laser processing [37-39]. However, they have not been implemented so far in DLIP.

In this present study, we monitored the heat accumulation during DLIP treatments of stainless steel and aluminum sheets. The surfaces of the samples are laser-treated by varying different process parameters such as laser power and repetition rate. An uncooled high-speed mid-wave infrared (MWIR) camera is utilized to detect the accumulated heat during the processing of the samples. The processed samples are characterized using confocal microscopy (CM) and the quality of the produced structures is correlated with the signal from the MWIR camera.

\section{Experimental procedure}

\subsection{Materials}

Commercial metallic sheets made of two different materials were used as a substrate for the laser experiments in this work. Stainless steel sheets (X5CrNi18-10, 1.4301) with a thickness of $0.8 \mathrm{~mm}$ and aluminum sheets (2024-T351) with $1 \mathrm{~mm}$ in thickness. They were electro-polished to obtain a smooth reflective surface. Prior to laser experiments, all samples were cleaned with ethanol to reduce any contaminations. The thermal conductivity of both $\mathrm{X} 5 \mathrm{CrNi} 18-10$ steel and 2024-T351 aluminum are 15 and $119 \mathrm{~W} / \mathrm{m} \cdot \mathrm{K}$, respectively $[40,41]$.

\subsection{DLIP laser setup}

The experimental setup used in this work is shown in Figure 1. The used laser process setup consists of a pulsed laser source combined with a modular DLIP head, which is mounted on a positioning stage system for moving the sample during the laser treatment.

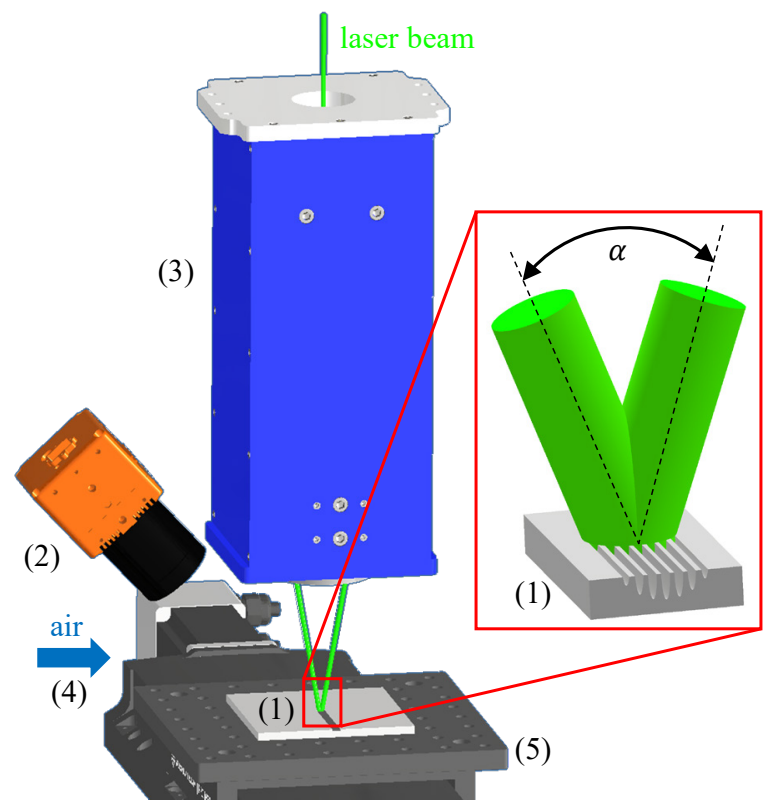

Fig. 1 Experimental setup for monitoring the heat accumulation during a two-beam DLIP treatment of metallic samples (1) with MWIR camera (2), DLIP head (3), a cross jet (4) and a positioning stage system (5). The two overlapped laser beams at an angle $\alpha$ are shown, resulting in a line-like structure pattern on the metallic sample (1).
The metallic samples were structured using a DLIP system (Fraunhofer IWS, Germany) based on a pulsed Nd:YVO4 laser (PX200-2-GFH, EdgeWave GmbH) emitting a wavelength of $\lambda=532 \mathrm{~nm}$ with a constant pulse duration of $\tau=12 \mathrm{ps}$. The laser provided repetition rates $f_{R}$ up to $100 \mathrm{kHz}$. The energy distribution of the outcoming beam was Gaussian shaped. A lens with a focal length of $f_{L}=100 \mathrm{~mm}$ superimposed the sub-beams with a certain overlapping angle $\alpha$ on the surface of the samples.

The thermal imaging system used to detect heat effects on the used material was a MWIR camera (TACHYON 16k, New Infrared Technologies, NIT) with an objective lens with a focal length $f_{C}=35 \mathrm{~mm}$. This camera allowed covering a wide range of temperatures $\left(100-2000{ }^{\circ} \mathrm{C}\right)$. The distance between the camera and the sample was adjusted to $100 \mathrm{~mm}$. The camera operated with a resolution of $64 \times 64$ pixels and a framerate of $1000 \mathrm{~Hz}$. It has to be mentioned, that due to the low resolution of the camera, it is not possible to measure the local temperatures at the different positions of the laser spot with the interference pattern. The acquisition time of the camera was $200 \mu \mathrm{s}$. The sensor of the camera had a spectral range between 1.0 and $5.0 \mu \mathrm{m}$ with its maximum sensitivity at the peak wavelength of $3.7 \mu \mathrm{m}$. In the experimental setup, the camera was located on the left side of the DLIP head at an angle of $45^{\circ}$ to the vertical in an off-axis configuration to the laser beam (see Figure 1). The used infrared camera has a silicon lens and a silicon window which filters the visible and near-infrared light including the reflection of the laser irradiation. The camera focused directly on the surface of the metallic samples in order to observe the laser treatment, especially the process zone. Due to the fact that the integration time of the camera $(200 \mu \mathrm{s})$ was much longer than the time between two pulses (e.g. $10 \mu \mathrm{s}$ at $100 \mathrm{kHz}$ ) as well as the pulse duration (10 ps), any synchronization of the camera with the laser process was performed. For protecting the camera and DLIP head from metallic dust, a cross jet with compressed air in combination with exhaustion was utilized. A two-axis positioning stage system (PR0165-300, Aerotech $\mathrm{GmbH})$ realized the movement of the metallic sample under the DLIP head in $x$ and $y$ directions.

To perform the experiments, the spot size $\phi$ was set to $140 \mu \mathrm{m}$ for the stainless steel sheets and $130 \mu \mathrm{m}$ for aluminum sheets. Table 1 summarizes the different process parameters used in the experiments for monitoring the thermal effects.

Table 1 Applied process parameter for the DLIP surface structuring of the metal samples

\begin{tabular}{ccc}
\hline$f_{R}$ in $\mathrm{kHz}$ & $o_{P}$ in $\%$ & $F$ in $\mathrm{J} / \mathrm{cm}^{2}$ \\
\hline 100 & $99 \ldots 99.67$ & $0.21 \ldots 0.69$ \\
80 & $99 \ldots 99.67$ & $0.18 \ldots 0.45$ \\
\hline
\end{tabular}

With this experimental setup, it was possible to produce line-like periodic structures with a defined spatial period of $\Lambda=4.5 \mu \mathrm{m}$. The strategy used for processing the samples is shown in Figure 2.

During the laser experiments, the overlap of the pulses $o_{P}$, the repetition rate of the laser $f_{R}$ and the laser power $P_{L}$ was varied according to Table 1 . By changing the laser power, the fluence $F$ was adjusted. The repetition rate 
also determined the speed of the positioning stage system. An area of $40 \times 5 \mathrm{~mm}^{2}$ was structured with each parameter set in parallel lines by moving the stage in y direction while the laser beam and the structuring process was active. Between the single lines, the stage moved in $\mathrm{x}$ and $\mathrm{y}$ direction. The principle is shown in Figure 2. After scanning each line over the material sample, the beam was moved laterally $140 \mu \mathrm{m}$ for stainless steel sheets and $130 \mu \mathrm{m}$ for aluminum (hatch distance $h$ ). Finally, the process was repeated at least 30 times until the total area to be structured was covered. All experiments were performed under ambient conditions and at room temperature.

\subsection{Surface characterization}

The topography of the processed samples was characterized by means of confocal microscopy with a 50x magnification objective (Sensofar S Neox, Spain). The optical and vertical resolutions of the objective were $170 \mathrm{~nm}$ and $3 \mathrm{~nm}$, respectively. The topography data were analyzed with the software MountainsMap ${ }^{\circledR} 7.4$.

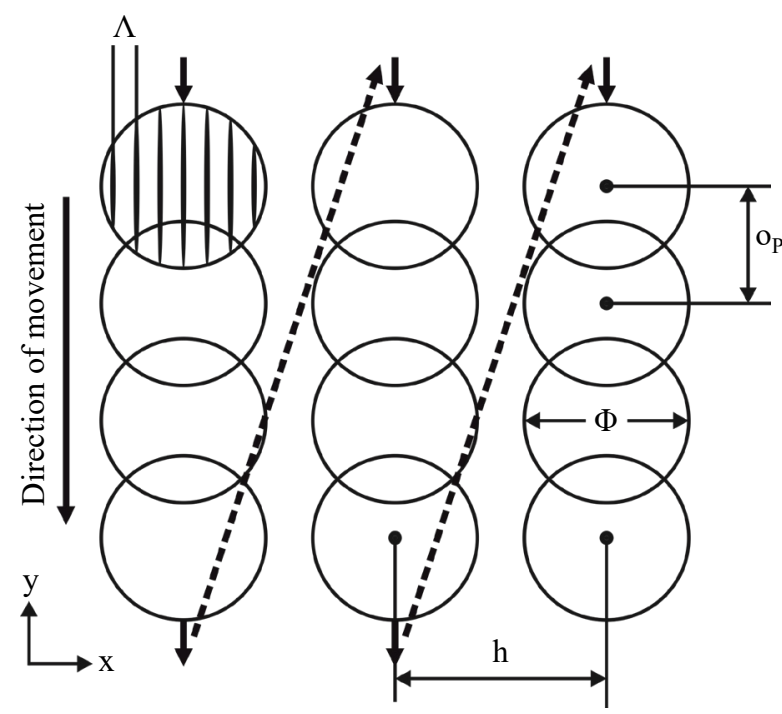

Fig. 2 Schematic sketch of the DLIP process with the resulting line-like pattern with process parameters: hatch distance $h$, spatial period $\Lambda$, overlap $o_{P}$, the diameter of the interference patterned area $\phi$ and the direction of movement of the positioning stage. Solid line: laser on, dashed line: laser off.

\section{Results and discussion}

\subsection{Thermal analyses strategy development}

For investigating the thermal effects occurring during the DLIP laser process, trenches of the $40 \times 5 \mathrm{~mm}^{2}$ area were processed. These areas were processed by using the strategy described in section 2.2 (see Figure 2).

Due to the proximity of the lines, which are processed continuously, different areas of interest were defined, which are the beginning, middle and end sections of the treated area. Moreover, the topography analyses were also performed at the $1^{\text {st }}, 15^{\text {th }}$ and $30^{\text {th }}$ line of each structured area. Figure 3 shows the schematic procedure and the strategy of the analysis. Note that each line in the figure represents the spot $(\sim 140 \mu \mathrm{m})$ containing the line-like interference pattern.

An example of confocal microscopy images of $100 \mu \mathrm{m} \times 180 \mu \mathrm{m}$ areas taken at the different above from the beginning, middle and end zone of the $1^{\text {st }}, 15^{\text {th }}$ and $30^{\text {th }}$ line as it is schematically shown in Figure 3. The confocal image corresponding to the beginning zone was taken after $2 \mathrm{~mm}$ from the starting position, while the images of the middle and end zones were taken after $20 \mathrm{~mm}$ and $38 \mathrm{~mm}$. These three single section images were used for analyzing the quality of the structure in each line (see Figure 3). In total, nine pictures were used for analyzing a structured area (of approximately $40 \times 5 \mathrm{~mm}^{2}$ ).

Undoubtedly, the amount of re-solidified material has a significant influence on the quality of the produced structure, since it can deteriorate the periodicity of the periodic structures as it has been already shown [42]. In consequence, the presence of excessive re-solidified material on top of the structures was a key criterion for classifying the resulting structures. The imperfection of the line-like structures was the deciding characteristic that appeared as rugged solidified material on or between the structures. This criterion was finally correlated with the recorded signal of the infrared camera during the laser experiments.

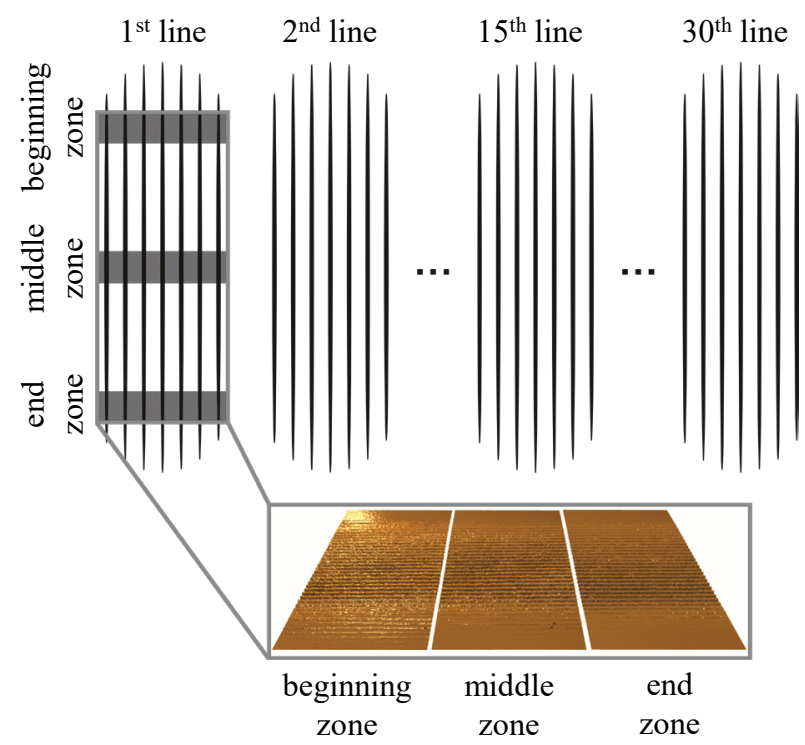

Fig. 3 Schematic representation of the resulting line-like patterns at the different processed lines. The regions corresponding to the beginning, middle and end zones are indicated as well as representative confocal images to analyze the quality of a line.

Figure 4 shows exemplarily the intensities of the detected infrared signal by the MWIR camera for stainless steel as a function of the line length $(40 \mathrm{~mm})$ for two processing conditions corresponding to fluences of $F=0.24 \mathrm{~J} / \mathrm{cm}^{2}$ and $0.59 \mathrm{~J} / \mathrm{cm}^{2}$.

The signal corresponds in both cases to the fifteenth line. The infrared signal of the line treated with $\mathrm{F}=0.59 \mathrm{~J} / \mathrm{cm}^{2}$ increases strongly at the beginning of the process. As more energy is introduced into the material, the generated heat might not be dissipated fast enough, which can be explained by the relative low thermal conductivity of the used stainless steel. In the middle position of the line $(\sim 20 \mathrm{~mm})$, the infrared signal drops slowly by $50 \%$. This level remains later constant until the end of the laser treatment. A possible explanation of this behavior could be that at this position an equilibrium between the absorbed energy from the laser and 
dissipated energy in the material occurs. Differently, the line treated with $F=0.24 \mathrm{~J} / \mathrm{cm}^{2}$ shows a much lower intensity level. During the whole laser process, the infrared signal stays on a constant level, which is about four times lower than the signal of the line processed at the higher fluence $\left(F=0.59 \mathrm{~J} / \mathrm{cm}^{2}\right)$. This means that higher infrared signals are obtained when increasing the laser fluence denoting possible differences in the heat conduction during the DLIP process.

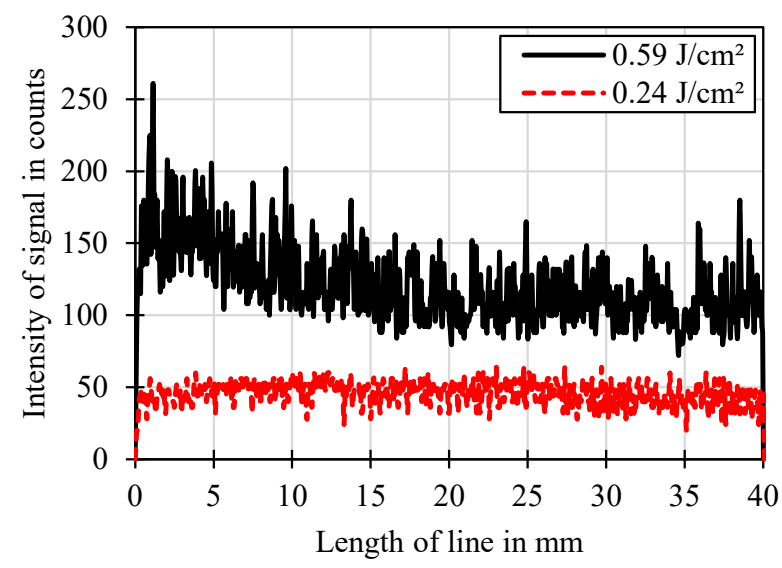

Fig. 4 MWIR intensity of the signal as a function of the process position for two different fluences (solid black line: $F=0.59 \mathrm{~J} / \mathrm{cm}^{2}$; red dashed line: $F=0.24 \mathrm{~J} / \mathrm{cm}^{2}$ ). Both lines were obtained with constant process parameters (Overlap $o_{P}=99.67 \%$, repetition rate $f_{R}=100 \mathrm{kHz}$ ).

The different behaviors in the detected MWIR signal are also reflected in the quality of the resulting surface structures due to the different used process parameters described above. This can be observed in Figure 5, where the confocal microscopy images for both process conditions are shown. The pictures correspond to the different zones, as explained above.

The surface topography of the sample that was processed with a fluence $F=0.24 \mathrm{~J} / \mathrm{cm}^{2}$ exhibits a homogenous structure without any significant amount of solidified material (Figure 5a). Clearly, the produced line-like pattern is visible at all positions. In comparison, the quality of the treated area with $F=0.59 \mathrm{~J} / \mathrm{cm}^{2}$ appears irregular and shows a significant excess of solidified material above the structured area. Moreover, these laser treated areas have a deteriorated pattern structure (Figure 5b). These two observed describe the surface quality criterion used in the rest of this study.

To find a correlation between the recorded infrared signal camera and the surface conditions, the difference between the maximum and minimum intensity value $\Delta \mathrm{i}$ of each curve was investigated. For example, in the case of the curves showed in Figure 4, this difference was $\Delta \mathrm{i}=56$ counts and $\Delta \mathrm{i}=256$ counts for $0.24 \mathrm{~J} / \mathrm{cm}^{2}$ and $0.59 \mathrm{~J} / \mathrm{cm}^{2}$, respectively.

\subsection{DLIP processing of stainless steel}

Structured areas with a line-like pattern were generated on stainless steel by varying the repetition rate, the overlap and the laser fluence. In Figure 6a-f, the differences between the maximum and minimum value of the intensity $\Delta i$ are illustrated as a function of the fluence $F$ for different repeti- tion rates and overlaps. Nearly all curves show a linear increase of the detected infrared signal as a function of the laser fluence. In general, with smaller overlaps (e.g. $99 \%$ ), the increase ratio for the intensity variation $\Delta i$ is lower compared to the reported data at higher overlaps. This trend occurs for both used repetition rates, $f_{R}=80 \mathrm{kHz}$ and $f_{R}=100 \mathrm{kHz}$.

Furthermore, there are almost no differences in both, the signal difference increase ratio as well as the absolute values of detected infrared signal for the investigated laser fluence range for overlaps of $99 \%$ or $99.5 \%$. Consequently, this can be attributed to any significant change in the surface temperature for these parameters. The difference of the intensity signal for the 1st, 15th and 30th lines is also marginal at all investigated fluences for the overlaps mentioned above. The small deviations might be a result of the noise of the camera. Hence, the resulting laser structured surfaces also do not show any significant differences in the performed confocal microscope analysis (not shown). This effect is independent of the set repetition rate.

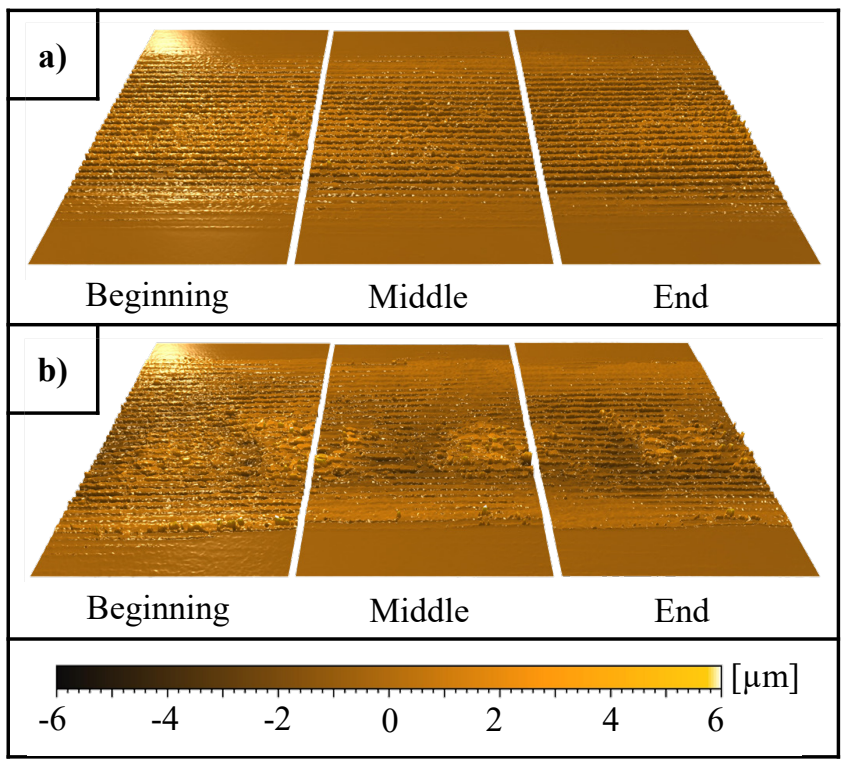

Fig. 5 Confocal microscope images of the structures produced at: (a) $F=0.24 \mathrm{~J} / \mathrm{cm}^{2}$, showing a homogenous pattern with no significant solidification, and at (b) $F=0.59 \mathrm{~J} / \mathrm{cm}^{2}$, showing a significant amount of solidification as well as a deteriorated pattern structure. All the pictures show the beginning, middle and end zones of the fifteenth line.

However, for high overlaps, a different behavior was observed. Firstly, the difference $\Delta i$ for the largest used overlap of $99.67 \%$ is significantly higher compared to the previous cases, for both repetition rates of $80 \mathrm{kHz}$ and $100 \mathrm{kHz}$. The curve with a parameter set of $f_{R}=100 \mathrm{kHz}$ and $o_{P}=99.67 \%$ has a unique curve progression (Figure $6 \mathrm{c}$ ). In particular, the linear increase in the difference signal difference $\Delta i$ is observed only for fluences below $F \leq 0.36 \mathrm{~J} / \mathrm{cm}^{2}$. At fluences above $0.47 \mathrm{~J} / \mathrm{cm}^{2}$, the curve starts to deviate from linear behavior. Also, significant differences in the infrared signal could be detected for the individual lines, 


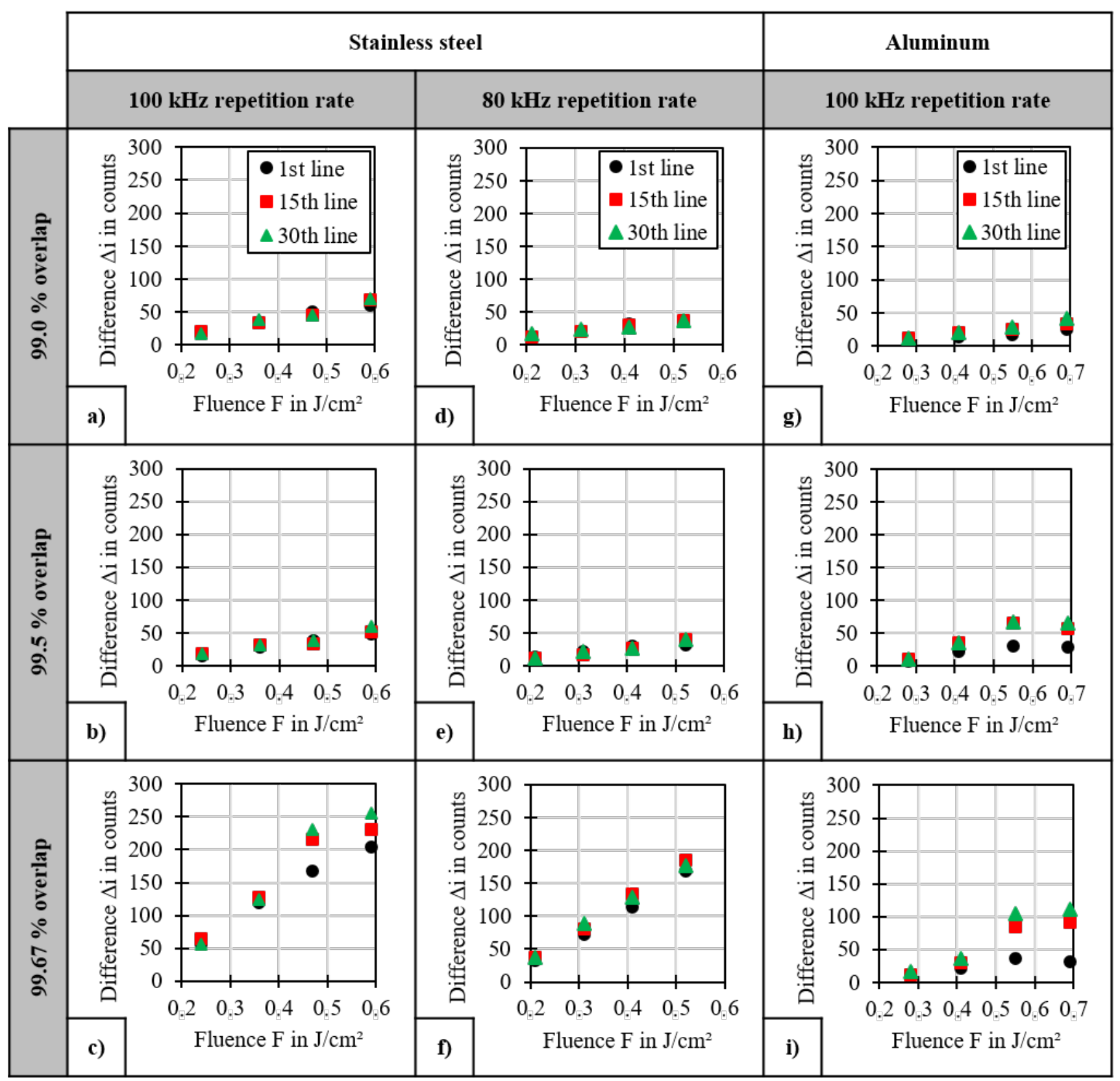

Fig. 6 Difference between the maximum and minimum value of the intensity $\Delta i$ as a function of the fluence $F$ by using a different repetition rates and overlaps. In the charts, the $1^{\text {st }}, 15^{\text {th }}$ and the $30^{\text {th }}$ lines are shown. Images (a-f) correspond to stainless steel: (a) $99.0 \%$ overlap, $100 \mathrm{kHz}$ repetition rate; (b) $99.5 \%$ overlap, $100 \mathrm{kHz}$ repetition rate; (c) $99.67 \%$ overlap, $100 \mathrm{kHz}$ repetition rate; (d) $99.0 \%$ overlap, $80 \mathrm{kHz}$ repetition rate; (e) $99.5 \%$ overlap, $80 \mathrm{kHz}$ repetition rate; (f) $99.67 \%$ overlap, $80 \mathrm{kHz}$ repetition rate. Images (g-i) correspond to aluminum: (e) $99 \%$ overlap, $100 \mathrm{kHz}$ repetition rate; (f) $99.5 \%$ overlap, $100 \mathrm{kHz}$ repetition rate; (i) $99.67 \%$ overlap, $100 \mathrm{kHz}$ repetition rate.

which means that the topography also within the same area can differ.

This can be seen in Figure 7, where the confocal microscope images of a $1^{\text {st }}, 15^{\text {th }}$ and $30^{\text {th }}$ line are presented. All the lines belong to the same structured area treated with one parameter set $\left(F=0.59 \mathrm{~J} / \mathrm{cm}^{2}, \quad o_{P}=99.67 \%\right.$ and $f_{R}=100 \mathrm{kHz}$ ). As the images show, the surface topography of every line is quite different. The amount of solidified material increases with the number of structured lines (see Figure 2). That is also reflected in the increment of the infrared signal occurring during the structure process of the specific lines (see Figure $6 \mathrm{c}, F=0.59 \mathrm{~J} / \mathrm{cm}^{2}$ ). Therefore, it can be concluded that the increment of remaining material after the laser process can be correlated with the increase of the surface temperature due to the accumulated heat in the material. The observed topography accordingly also explains the increase in the measured infrared signal, since higher temperatures results in a larger amount of counts captured by the camera. 


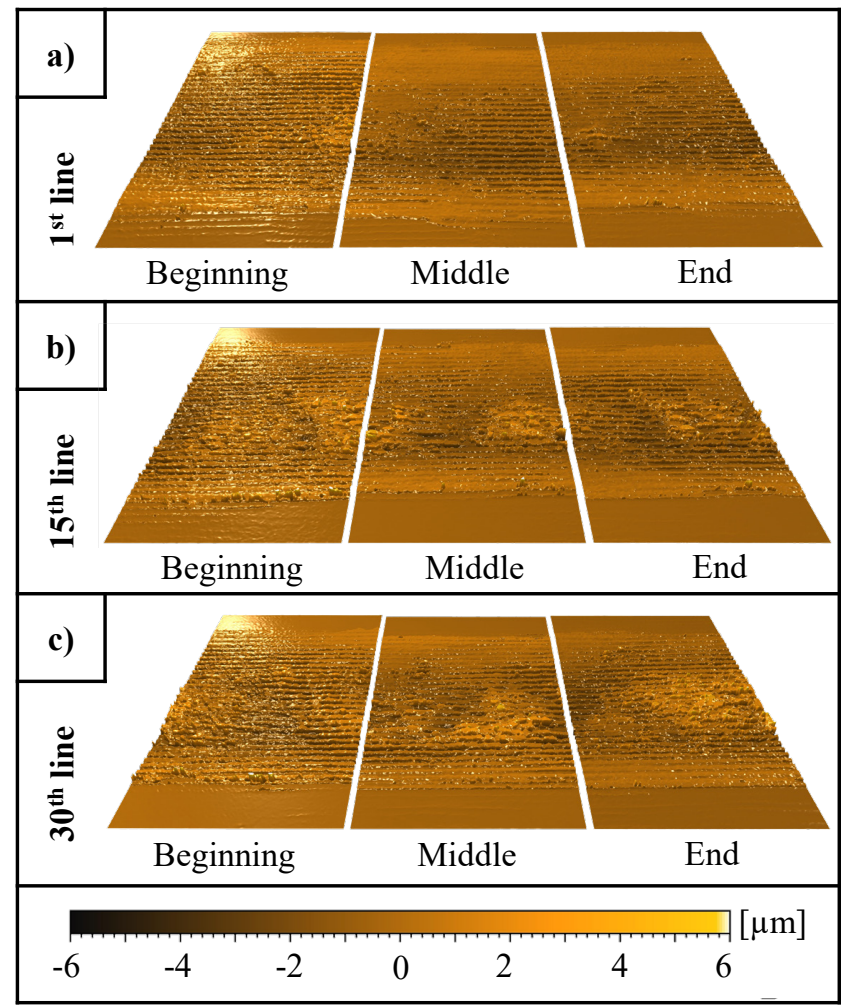

Fig. 7 Confocal microscope images of the specific lines produced on stainless steel at $F=0.59 \mathrm{~J} / \mathrm{cm}^{2}, o_{P}=99.67 \%$ and $f_{R}=100 \mathrm{kHz}$. (a) $1^{\text {st }}$ line; (b) $15^{\text {th }}$ line and (c) $30^{\text {th }}$ line.

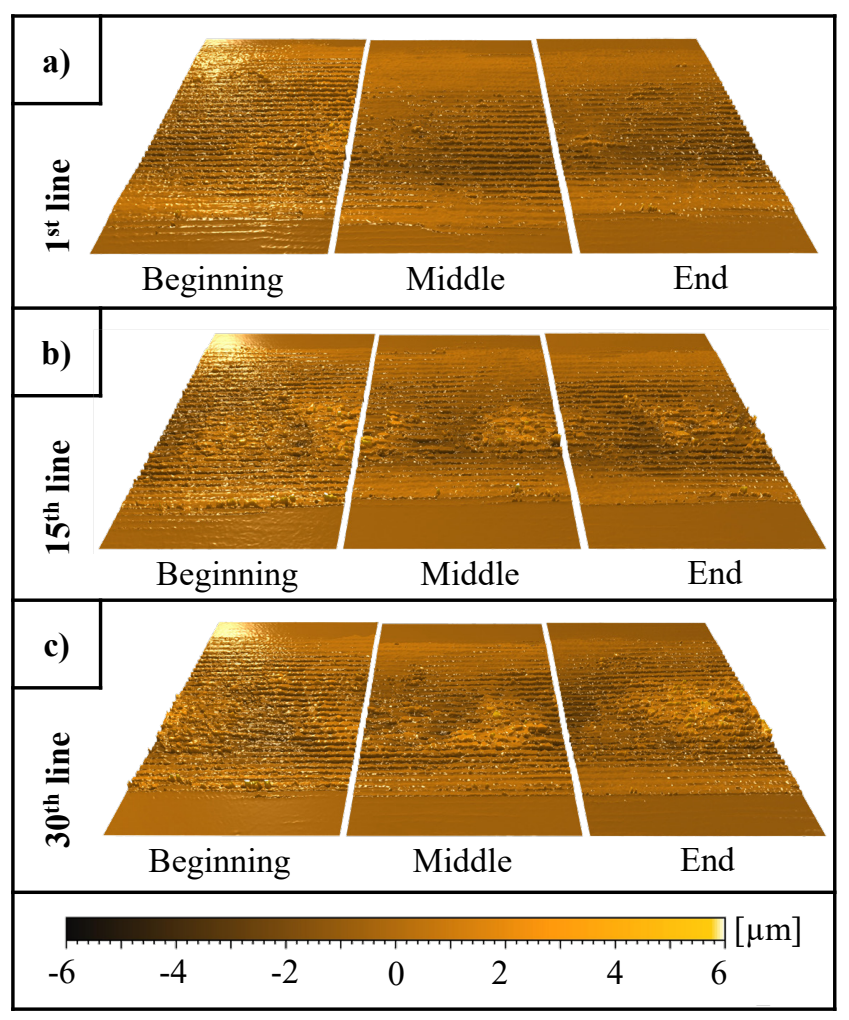

Fig. 8 Confocal microscope images of the specific lines produced on aluminum at $F=0.69 \mathrm{~J} / \mathrm{cm}^{2}, o_{P}=99.67 \%$ and $f_{R}=100 \mathrm{kHz}$. (a) $1^{\text {st }}$ line; (b) $15^{\text {th }}$ line and (c) $30^{\text {th }}$ line.

\subsection{DLIP processing of aluminum}

The experiments with aluminum sheets were also conducted with different overlaps and a fixed repetition rate of $100 \mathrm{kHz}$. Figure 6g-i shows the difference between the maximum and minimum values of the infrared intensity $\Delta i$ as a function of the laser fluence $F$. In this case, the increase of the measured signal difference intensity shows a linear tendency, but only for the lowest used pulse to pulse overlap ( $99 \%$, see Figure 6a). With higher overlaps, the intensity of the detected infrared radiation also increases but showing a non-linear evolution.

Also, the difference between the intensity of the $1^{\text {st }}, 15^{\text {th }}$ and $30^{\text {th }}$ lines increases with higher fluences and overlaps. This behavior was reported in stainless steel only for the parameter set of fluences above $0.47 \mathrm{~J} / \mathrm{cm}^{2}, 100 \mathrm{kHz}$ and $99.67 \%$ overlap.

Figure 8 shows exemplarily for this material, the confocal microscope images of the $1^{\text {st }}, 15^{\text {th }}$ and $30^{\text {th }}$ lines. The images indicate different surface qualities of the lines fabricated during a DLIP treatment of aluminum (at $F=0.69 \mathrm{~J} / \mathrm{cm}^{2}, o_{P}=99.67 \%$ and $f_{R}=100 \mathrm{kHz}$ ). The appearance of solidified material on top of the structured pattern also differs in this case according to the quantity of structured lines. This behavior is similar to the case of the stainless steel substrates also treated at high overlap and laser fluence (see section 3.2).

Therefore, also for aluminum, it can be assumed that due to the high amount of energy provided by the laser system when using high repetition rates as well as high fluences, with each line, the added energy into the material has not enough time for heat dissipation and in consequence a temperature increase with increasing the number of lines occurs. Consequently, the infrared signal captured by the camera also increases.

\subsection{Evaluation of a criterion for determining surface quality using MWIR signal}

In order to evaluate a possible criterion indicating excess solidified material during the DLIP process, the measured infrared radiation differences for both stainless steel and aluminum samples are shown in Figure 9, as a function of the repetition rate, laser fluence and overlap. The fields highlighted with green horizontal lines indicate surface conditions without any excess solidified material. Contrary to that, the surface quality of fields with red dots shows an excessive amount of solidified material on the treated metallic samples.

The reported values indicate a general characteristic of infrared signal differences occurring at a DLIP process for both stainless steel and aluminum. In the case of stainless steel, all measured topographies with a satisfactory surface quality presented MWIR signal values smaller than $\sim 38$ counts. For aluminum, the same was observed for values smaller than 18 counts. With exceeding these limits, the surface condition for all samples exhibits an excessive amount of solidified material on the surfaces.

During the laser treatment of the aluminum samples, the reached signal intensity $\Delta \mathrm{i}$ with a certain fluence was lower compared to the signal intensity of stainless steel. This condition was independent of the set fluence. The thermal conductivity of stainless steel is eight times smaller than the value of aluminum $(15 \mathrm{~W} / \mathrm{m} \cdot \mathrm{K}$ and $119 \mathrm{~W} / \mathrm{m} \cdot \mathrm{K}$ for steel and aluminum, respectively). Clearly, for the metal with the 


\begin{tabular}{|c|c|c|c|c|c|c|c|c|c|}
\hline \multirow[b]{3}{*}{$\begin{array}{l}\text { Fluence } \\
\text { in } \mathrm{J} / \mathrm{cm}^{2}\end{array}$} & \multicolumn{3}{|c|}{$99 \%$ overlap } & \multicolumn{3}{|c|}{$99.5 \%$ overlap } & \multicolumn{3}{|c|}{$99.67 \%$ overlap } \\
\hline & \multicolumn{2}{|c|}{ Stainless steel } & \multirow{2}{*}{$\begin{array}{c}\mathrm{Al} \\
100 \mathrm{kHz}\end{array}$} & \multicolumn{2}{|c|}{ Stainless steel } & \multirow{2}{*}{$\begin{array}{c}\mathrm{Al} \\
100 \mathrm{kHz}\end{array}$} & \multicolumn{2}{|c|}{ Stainless steel } & \multirow{2}{*}{$\begin{array}{c}\mathrm{Al} \\
100 \mathrm{kHz}\end{array}$} \\
\hline & $100 \mathrm{kHz}$ & $80 \mathrm{kHz}$ & & $100 \mathrm{kHz}$ & $80 \mathrm{kHz}$ & & $100 \mathrm{kHz}$ & $80 \mathrm{kHz}$ & \\
\hline $0.2-0.3$ & 20 & E14 & 12 & $18 \bar{z}$ & E12 & $E 10^{z}$ & $\bar{E} 60^{z}$ & E36 & $=13$ \\
\hline $0.3-0.4$ & 35 垔 & 르르를 & - & 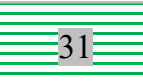 & 正20 & - & $y_{124}$ & ${ }^{4} 81$ & - \\
\hline $0.4-0.5$ & 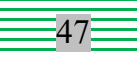 & $=30$ 五 & 18 & 37 & E27 & & 204 & 3118 & 335,4 \\
\hline $0.5-0.6$ & 66 & $\bar{E} 38 \overline{\underline{ }}$ & 24 & $\overline{\underline{\underline{ }}} 53$ 五 & 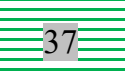 & 54 & 239 & 177 & 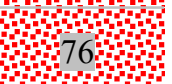 \\
\hline $0.6-0.7$ & - & - & ${ }^{2} 33{ }^{3}$ & - & - & Hit & - & - & Ply \\
\hline
\end{tabular}

Fig. 9 Measured infrared signal intensity $\Delta i$ as function of the process parameters for both stainless steel and aluminum samples. The color in the diagram represents two obtained surface conditions: no significant solidified material on the surface (green horizontal lines); excessive solidified material on the surface (red squares).

highest thermal conductivity, the produced heat during the laser process can be faster evacuated from the process zone to the surrounding material much efficiently and faster.

This feature might have caused the reported differences between the intensity of detected infrared radiation for stainless steel and aluminum sheets. Another possibility can be related to the melting and vaporization temperatures for aluminum compared to stainless steel. Thus, DLIP ablation process at aluminum is conducted at a lower temperature, which can also explain the lower MWIR signal for this material. Other effects that have an influence on the recorded infrared intensity are the emissivity of the used materials as well as the surface roughness which is modified during the laser process [43-45]. These parameters will be evaluated in the future.

\section{Conclusions}

The influence of thermal effects on the surface quality for DLIP treated stainless steel and aluminum samples were discussed in this work. The objective was to evaluate if using an infrared camera, it is possible to detect in-line instabilities affecting the surface quality of the produced periodic linelike patterns negatively.

The MWIR camera could satisfactorily detect the infrared radiation caused by the DLIP process in the metallic samples. The obtained results revealed a correlation between the recorded infrared signal by the MWIR camera and the surface quality. Detailed investigation showed an impact of the thermal effects on the quality of the surfaces and the amount of solidified material on the line-like pattern. It was observed that these physical processes were influenced by the used process parameter.

Threshold values corresponding to the intensity difference of the infrared signal detected by the MWIR camera permitted to classify the obtained surface conditions. The intensity limit for aluminum sheets was around twice times lower (18) compared to the determined value for stainless steel (38). The thermal conductivity of both materials as well as the melting and vaporization temperatures, could explain these different investigated values. In the case of exceeding this threshold limit, the deterioration of the line-like surface pattern was visible, and even differences within the same areas as well as during the process could be detected using the here presented method.

Future work will be focused on the development of a simulation model based on DLIP to compare the here presented results with the thermal history of metallic samples under different process conditions.

\section{Acknowledgments}

This project has received funding from the European Union's Horizon 2020 research and innovation programme under Grant Agreement No. 825132. It is an initiative of the Photonics Public Private Partnership. www.photonics21.org Any dissemination of results must indicate that it reflects only the author's view and that the EU is not responsible for any use that may be made of the information it contains.

\section{References}

[1] A. A. Tyuftin and J. P. Kerry: Food Packag. Shelf Life, 24, (2020) 100475.

[2] F. Guo and Z. Guo: RSC Adv., 6, (2016) 36623.

[3] T. B. H. Schroeder, J. Houghtaling, B. D. Wilts, and M. Mayer: Adv. Mater., 30, (2018) 1705322.

[4] C. Zhang, D. A. Mcadams, and J. C. Grunlan: Adv. Mater., 28, (2016) 6292.

[5] G. Liu, Z. Yuan, Z. Qiu, S. Feng, Y. Xie, D. Leng, and X. Tian: Ocean Eng., 199, (2020) 106962.

[6] C. Neinhuis: Ann. Bot., 79, (1997) 667.

[7] Z. Yang, C. Zhu, N. Zheng, D. Le, and J. Zhou: Materials, 11, (2018) 2210.

[8] R. S. S. Raja, P. Selvakumar, and P. D. Babu: J Mech Sci Technol, 34, (2020) 1667.

[9] S. A. Jalil, B. Lai, M. ElKabbash, J. Zhang, E. M. Garcell, S. Singh, and C. Guo: Light Sci. Appl., 9, (2020) 14. 
[10] M. Petkovšek, M. Hočevar, and P. Gregorčič: Ultrason Sonochem, 67, (2020) 105126.

[11] C. Li, L. Yang, N. Liu, Y. Yang, J. Zhao, P. Yang, and G. Cheng: Surf. Coat. Technol., 388, (2020) 125594.

[12] E. Liu, H. J. Lee, and X. Lu: Appl. Sci., 10, (2020) 2678.

[13] D. W. Müller, T. Fox, P. G. Grützmacher, S. Suarez, and F. Mücklich: Sci. Rep., 10, (2020) 3647.

[14] A. F. Lasagni, D. Benke, T. Kunze, M. Bieda, S. Eckhardt, T. Roch, D. Langheinrich, and J. Berger: J. Laser Micro/Nanoengin., 10, (2015) 340.

[15] S. Indrišiūnas, B. Voisiat, M. Gedvilas, and G. Račiukaitis: J. Laser Appl., 29, (2017) 011501.

[16] A. F. Lasagni, P. Shao, J. L. Hendricks, C. M. Shaw, D. C. Martin, and S. Das: Appl. Surf. Sci., 256, (2010) 1708.

[17] Y. Li and M. Hong: Laser Photonics Rev., 14, (2020) 1900062

[18] Y. Lin, M. H. Hong, T. C. Chong, C. S. Lim, G. X. Chen, L. S. Tan, Z. B. Wang, and L. P. Shi: Appl. Phys. Lett., 89, (2006) 041108.

[19] Y. Zhou, M. H. Hong, J. Y. H. Fuh, L. Lu, and B. S. Lukiyanchuk: Phys. Scr., 129, (2007) 35.

[20] W. Zhao, H. Liu, X. Shen, L. Wang, and X. Mei: Materials, 13, (2019) 31.

[21] D. Metzner, P. Lickschat, and S. Weißmantel: Appl. Phys. A, 125, (2019) 462.

[22] K.-H. Leitz, B. Redlingshöfer, Y. Reg, A. Otto, and M. Schmidt: Phys. Procedia, 12, (2011) 230.

[23] F. Bauer, A. Michalowski, T. Kiedrowski, and S. Nolte: Opt. Express, 23, (2015) 1035.

[24] R. Linares, G. Vergara, R. Gutiérrez, C. Fernández, V. Villamayor, L. Gómez, M. González-Camino, A. Baldasano, G. Castro, R. Arias, Y. Lapido, J. Rodríguez, and P. Romero: Proc. SPIE, 9485, (2015) 948514.

[25] A. Schmailzl, S. Steger, and S. Hierl: Laser Tech. J., 12, (2015) 34.

[26] X. Xiao, X. Liu, M. Cheng, and L. Song: J Mater Process Technol, 277, (2020) 116409.

[27] J. Ma, F. Kong, and R. Kovacevic: Mater. Des., 36, (2012) 348.

[28] A. Schmailzl, J. Käsbauer, J. Martan, P. Honnerová, F. Schäfer, M. Fichtl, T. Lehrer, J. Tesař, M. Honner, and S. Hierl: Int. J. Heat Mass Transf., 146, (2020) 118814.

[29] H. Xu, X. Guo, Y. Lei, J. Lin, H. Fu, R. Xiao, T. Huang, and Y. C. Shin: Opt Laser Technol, 119, (2019) 105583.

[30] M. Y. Kayacan and N. Y1lmaz: Measurement, 160, (2020) 107825.

[31] A. Chabot, M. Rauch, and J.-Y. Hascoët: Weld World, 63, (2019) 759.

[32] L. Zheng, Q. Zhang, H. Cao, W. Wu, H. Ma, X. Ding, J. Yang, X. Duan, and S. Fan: Mater. Des., 183, (2019) 108110.

[33] P. M. Cordero, J. Mireles, S. Ridwan, and R. B. Wicker: Prog Addit Manuf, 2, (2017) 1.

[34] J. A. Mitchell, T. A. Ivanoff, D. Dagel, J. D. Madison, and B. Jared: Addit Manuf, 31, (2020) 100946.
[35] I. Yadroitsev, P. Krakhmalev, and I. Yadroitsava: J. Alloys Compd., 583, (2014) 404.

[36] R. Weber, T. Graf, P. Berger, V. Onuseit, M. Wiedenmann, C. Freitag, and A. Feuer: Opt. Express, 22, (2014) 11312.

[37] J. H. Pak, S. Jeong, T. Lim, and S. Ju: Adv. Optical Mater., 8, (2020) 1901706.

[38] D. V. Tran, Y. C. Lam, H. Y. Zheng, B. S. Wong, and D. E. Hardt: Appl. Surf. Sci., 253, (2007) 7290.

[39] D. V. Tran, Y. C. Lam, B. S. Wong, H. Y. Zheng, and D. E. Hardt: Opt. Express, 14, (2006) 9261.

[40] S. Ertl: Investigation on the production and use of microtechnically manufactured diamond tools, Dissertation, University of Freiburg, (2003).

[41] R. C. Batra and C. H. Kim: Int. J. Plast., 8, (1992) 425.

[42] A. I. Aguilar-Morales, S. Alamri, T. Kunze, and A. F. Lasagni: Opt Laser Technol, 107, (2018) 216.

[43] C.-D. Wen and I. Mudawar: Int. J. Heat Mass Transf., 49, (2006) 4279.

[44] S. Taylor, J. B. Wright, E. C. Forrest, B. Jared, J. Koepke, and J. Beaman: Int. Commun. Heat Mass Transf., 115, (2020) 104614.

[45] T. L. Bergman and A. S. Lavine: Fundamentals of Heat and Mass Transfer, (2017).

(Received: June 19, 2020, Accepted: September 15, 2020) 